QUADERNS DE FILOSOFIA VOL. V NÚM. 2 (20I 8): 49-7I

eISSN: 234I-3042 DOI: IO.7203/QFIA.5.2.I2909

María Dolores García-Arnaldos

Universidade de Santiago de Compostela

\title{
¿Intuición o confianza racional?
}

Intuition or rational trust?

Recibido: 26/7/2018 . Aceptado: 15/10/2018

Resumen: Según la concepción tradicional, la justificación de las creencias lógicas básicas —entendida tanto inferencial como no-inferencialmente- no logra evitar ni la circularidad, ni la regresión al infinito. Justificar reglas básicas lógicas inferencialmente conlleva usar principios lógicos con lo que se genera un círculo vicioso. Apelar a fuentes básicas como la intuición, no sortea todas las dificultades. Argumentaré que es preciso recurrir a una "habilitación" (entitlement), una sub-clase dentro de las garantías epistémicas. Si además aceptamos que intuir es algo que hacemos y no algo que nos pasa, podemos sostener que la intuición, de hecho, se apoya en una confianza racional.

Abstract: According to the traditional conception, the justification of basic logical beliefs — understood both inferentially and non-inferentially - cannot avoid either circularity or regression to infinity. To justify logical basic rules inferentially involves using logical principles, so a vicious circle is generated. Appealing to basic sources such as intuition, does not avoid such problems. I will argue that it is necessary to resort to an entitlement, a sub-class within the epistemic warrants. If we also accept that intuiting is something that we do and not something that happens to us, we can sustain that intuition, indeed, is based on a rational trust.

Palabras clave: justificación, conocimiento lógico, garantía racional, intuición, habilitación.

Keywords: justification, logical knowledge, rational warrant, intuition, entitlement. 


\section{§I. INTRODUCCIÓN}

1 ormalmente, cuando razonamos siguiendo una regla lógica como el mocreemos que, si las premisas son verdaderas, la conclusión será necesariamente verdadera. Por ejemplo, si razonamos:

Si tengo el virus de la gripe, entonces he pillado una enfermedad infecciosa Tengo la gripe,

Luego, tengo una enfermedad infecciosa.

no nos cabe duda de que, si las premisas son verdaderas, la conclusión lo es.

La dificultad se plantea a la hora de responder a la pregunta: cómo sabemos que ese principio fundamental de razonamiento es válido. Es decir, cómo justificamos el conocimiento de la validez de un principio lógico, o de una verdad lógica como el MPP.

Sin agotar las posibilidades, las respuestas al problema de la justificación del conocimiento lógico que analizaremos aquí son, sobre todo, aquellas que han dado una solución no-inferencial.

La justificación no-inferencial de las creencias en el carácter correcto o preservador de verdad de una regla de inferencia puede ser entendida como una facultad quasi-perceptiva o intuición lógica o como una justificación del conocimiento lógico apelando a creencias razonables por defecto ${ }^{1}$ (FIELD 2000). Argumentaré que, ante las dificultades que se les presentan a las teorías basadas en la intuición, es preciso recurrir a una justificación en sentido débil o "habilitación" (entitlement), una sub-clase dentro de las garantías epistémicas. En última instancia, intuir es algo que hacemos y no algo que nos pasa. Sostendré que la intuición, así entendida, se apoya en una confianza racional.

\section{\$2. CONOCIMIENTO LÓGICO BÁSICO: JUSTIFICACIÓN NO-INFERENCIAL}

Cuando razonamos siguiendo la regla lógica del MPP, nos parece evidente que, si las premisas son verdaderas, la conclusión lo es, como hemos indicado arriba. El MPP es una regla tan básica que, de pronto, nos puede parecer que no es posible justificar una creencia tan fundamental o que es auto-evidente. Frege (I964; I979), por ejemplo, apela a la auto-evidencia (self-evidence) para

\footnotetext{
${ }^{1}$ En este artículo no abordamos el análisis de las teorías de la justificación por defecto.
} 
la justificación del conocimiento lógico. Para explicar el carácter auto justificativo de los axiomas lógicos o de las reglas de inferencia básicas Frege (i979, 175) considera que, lo que fundamenta (grounds) una proposición básica es ella misma ${ }^{2}$. En la visión tradicional, la auto-evidencia era un modo de caracterizar el conocimiento a priori. Leibniz, por ejemplo, consideraba que los axiomas eran evidentes ex terminis: "(...) esas proposiciones [axiomas] son evidentes ex terminis, en cuanto se entiendan sus términos, de modo que estaban persuadidos de que la fuerza de la convicción se fundamentaba en la inteligencia de los términos, es decir, en la relación entre sus ideas" (Leibniz I983, 488).

No obstante, a la hora de dar cuenta del conocimiento lógico básico, la intuición ha sido la fuente de conocimiento básico a priori más ampliamente aceptada porque evita el círculo vicioso en el que inevitablemente se cae al establecer que los principios de la lógica se justifican a partir de inferencias. Asumir que se adquirieren inferencialmente supone que uno sabe previamente que dichos principios o reglas de inferencia son válidos, con lo cual se genera circularidad (Bermejo 20 I I). Ahora bien, es necesario explicar en qué consiste una facultad quasi-perceptiva como la intuición y cómo esta justifica las creencias lógicas básicas. Clásicamente, ha sido presentada como la facultad por la que directamente captamos las propiedades necesarias, de tal modo que, si una inferencia proporcionaba conocimiento a priori, era debido a una evidencia racional directa. Ahora bien, según Boghossian (2000, 231), nadie ha sabido explicar en qué consiste esa facultad.

Si optamos por la otra fuente alternativa de conocimiento básico (la experiencia), encontramos teorías de la justificación del conocimiento lógico como la quineana. Según este enfoque, cualquier afirmación de conocimiento de principios lógicos básicos como la regla del MPP, así como de conocimiento de las leyes de la ciencia empírica, y cualquier regla, sea lógica o no, puede ser revisada, en principio, cuando el sistema de creencias entra en conflicto con la experiencia. La propuesta de Quine (1936; 1963) es una alternativa al conocimiento intuitivo de las leyes fundamentales de inferencia y al conocimiento inferencial a priori, de tal modo que el conocimiento de las leyes lógicas básicas sería parte de nuestro conocimiento empírico. El problema del conocimiento empírico es que la adquisición de conocimiento a través de inferencias exige el conocimiento de la validez de los principios de inferencia que se estén usando y, por ello, cae también en la circularidad (BOGHOSSIAN 2000; WRIGHT

${ }^{2}$ Así también lo indica y comenta Burge: "Los axiomas no son demostrables, ya que no es posible una prueba genuina, ninguna justificación de las verdades más básicas. Como son autoevidentes, no necesitan pruebas. Son auto-evidentes porque su justificación se encuentra en sus propios contenidos. Entender el contenido de un axioma es suficiente para garantizar que uno lo crea" (BuRge 2005, 337-8). [La traducción, cuando no se indique lo contrario, es mía.] 
2004B). Desde el punto de vista fundacionista, se sostiene entonces que la circularidad en la que incurren las justificaciones empíricas o a posteriori es tan rechazable como la que se presenta en el caso de las teorías a priori acerca del conocimiento lógico básico (MartíneZ-VIDAL 2008).

Hay otros enfoques, como el escepticismo, desde los que se argumenta que, aunque sería necesaria la justificación, no es posible justificar inferencias básicas como la regla del MPP. Otros planteamientos, como el expresivismo ${ }^{3}$ (Gibbard I990), no admiten que haya hechos lógicos y se engloban por ello en el no-factualismo respecto de la lógica, postura desde la que se sostiene, en términos generales, que las reglas lógicas no pueden justificarse ni es necesaria justificación alguna. Otra teoría que ha dado una respuesta positiva pero inferencial a partir de justificaciones regla-circulares es la de Boghossian (2000), teoría en la que aquí no entraremos.

\section{\$3. QUÉ ES UNA INTUICIÓN}

En las teorías de la justificación del conocimiento a priorí, la intuición se entiende de diversos modos. Para BonJour ( 1998) por ejemplo, consiste fundamentalmente en un insight (captación interna, aprehensión) racional. Otras teorías apelan a un tipo de creencias o a un estado quasi-perceptivo, como las de Sosa (2007), Bengson (20 I 5), Bealer (2000) — que en realidad es catalogado como una postura híbrida entre la concepción que se basa en la intuición y la que se basa en el significado_-, entre otros. Ahora bien, es necesario explicar en qué consiste una facultad quasi-perceptiva y cómo esta facultad justifica las creencias lógicas básicas.

Una presentación clásica de la concepción basada en la intuición la encontramos ya en Aristóteles o Descartes ${ }^{6}$. Según Aristóteles, la "percepción

${ }^{3}$ Dos de los representantes del expresivismo semántico son Sellars (1963) y Brandom (2000). Cuando el enfoque expresivista se aplica a nociones lógicas es llamado "expresivismo lógico".

${ }^{4}$ En el debate contemporáneo en torno a la justificación del conocimiento a priori — partimos del supuesto de que el conocimiento lógico es un tipo de conocimiento a priori-, las dos concepciones principales son aquellas basadas en la intuición y las basadas en el significado (DoGRAMACI 20I2), opción esta última en la que aquí no entraremos.

${ }^{5}$ BonJour prefiere usar el término 'a priori insight' al término 'intuición'.

${ }^{6}$ Descartes sostiene que las dos fuentes primeras de todo conocimiento son la intuición y la deducción: "Así todos vemos por intuición que existimos, que pensamos, que un triángulo está formado por tres líneas, que un globo no tiene más que una superficie, y otras verdades semejantes [...]. La intuición debe llevar consigo la certeza no sólo en las enunciaciones, sino en toda clase de razonamientos" (Reglas para la dirección del espiritu (I70I), Reglas III). Una deducción del tipo que presenta Descartes necesita de premisas universales, lo cual se convirtió en el talón de Aquiles del racionalismo y se prestó a la crítica de Hume, no se puede intuir una conexión necesaria de la realidad (Hume 1740, An Abstract of a Treatise, \$35-41. Y también (I739-I740), Treatise \$53-55). 
intelectiva" es el estado cognitivo que nos permite adquirir los primeros principios. Esta noción de aprehensión directa es semejante a la que Gödel, en el siglo $\mathrm{xx}$, utiliza para referirse a cómo conocemos o captamos los axiomas de la teoría de conjuntos?: "[...] tenemos algo así como una percepción de los objetos de la teoría de conjuntos, como se ve en el hecho de que los axiomas se imponen sobre nosotros como siendo verdaderos. No veo ninguna razón por la que deberíamos tener menos confianza en este tipo de percepción, es decir, en la intuición matemática que, en la percepción sensorial, [...]" (GöDEL I964, 271).

Actualmente, las teorías que se basan en la intuición han cobrado un renovado vigor al presentarse en forma de teorías explicativas unificadas de la justificación epistémica en las que se establece una cierta analogía: así como las experiencias perceptivas explican la justificación empírica, las intuiciones explican la justificación a priori (GARCía-CARPINTERo 2005).

Por otra parte, el término "juicio intuitivo" es usado en la práctica filosófica habitual como contraposición al término "juicio contra-intuitivo". Aunque también se usa de un modo especial en la filosofía contemporánea, en el sentido de que las intuiciones son acerca de casos que pueden ser hipotéticos, como los casos Gettier, por ejemplo. En cuanto al modo de entender lo que son, Bealer, como veremos, denomina a la intuición "parecer intelectual": "una actitud proposicional sui generis, irreductible [...] que ocurre de forma episódica” (BEALER I998, 207). BonJour (200I; 2005), en cambio, mantiene que las intuiciones son un tipo de estado mental semejante a las experiencias perceptivas.

Ahora bien, las teorías del a priori basadas en la intuición se encuentran con dos problemas, al menos, a los que no logran dar una salida satisfactoria. En primer lugar, cómo la intuición puede explicar la justificación inferencial. En segundo lugar, si se entiende la intuición como "parecer", cómo sabemos que un cierto "parecer" es o no fiable, cómo da cuenta del fiabilismo. BonJour y Bealer han tratado de buscar soluciones a ambos problemas.

\section{\$3.1 BonJour: el insight racional}

La propuesta de BonJour (1998) se enmarca en el racionalismo — aunque desde un fundacionismo racionalista moderado $^{8}$ dado que admite la fali-

${ }^{7}$ En matemáticas se ha explotado mucho la cuestión de la intuición y de las facultades quasiperceptivas de la intuición matemática. Además de Gödel, también Maddy y Parsons, entre otros, han discutido esto. Martínez-Vidal analiza, teniendo en cuenta estos autores, si la intuición proporciona fundamento epistémico concluyendo que ninguna de las propuestas de dichos autores proporciona una explicación aceptable de la intuición (MARTínEZ-VIDAL 2016).

${ }^{8}$ Otro tipo de fundacionismo moderado es el que admite, por otra parte, que haya ciertas creencias prima facie (AUDI 200I, 20 y ss). 
bilidad (BonJour I985, 193)—. Para BonJour', las intuiciones son evidencias racionales que posibilitan contrastar la verdad o la falsedad de la proposición, es decir, proporcionan justificación epistémica evidencial. En particular, el insight racional es fuente genuina de justificación:

[...] cuando considero cuidadosa y reflexivamente la proposición (o inferencia) en cuestión, simplemente puedo ver o captar o comprender que la proposición es necesaria, que debe ser verdadera en cualquier mundo o situación posible (o, alternativamente, que la conclusión de la inferencia debe ser verdadera si las premisas son verdaderas). Tal insight racional, [...] no parece depender en general de ningún tipo particular de criterio o de ningún otro proceso discursivo o de razonamiento, sino que es directo e inmediato [...] (BonJour I998, 106-7)

La captación de la necesidad de la proposición se produce a partir de una atenta reflexión, pero el momento de la captación o aprehensión es directo e inmediato. No la reflexión sino la captación, el ver la necesidad de la proposición, es el conocimiento directo e inmediato (y por eso básico) que BonJour denomina insight. Del insight racional resultan las razones a priori. Una razón a priori (BonJour 2005, 98) es aquella fuerza racional que no deriva de la experiencia ${ }^{10}$ directa (percepción) o indirecta (por ser una inferencia deductiva, inductiva, o de cualquier otra naturaleza, a partir de premisas derivadas de la experiencia). No obstante, tener una razón a priori requiere entender la afirmación para la cual es una razón, y la experiencia puede ser necesaria para entender esa afirmación. La idea de razón a priori, entendida en este sentido, no implica que no haya experiencias que puedan servir de base para una determinada proposición, o que tales experiencias no puedan anular (de manera más o menos concluyente) la razón a priori en cuestión. Tampoco significa que la razón a priori haga que la afirmación resulte ser cierta o infalible.

Por otra parte, una razón a priori resulta de la percepción intelectual (insight) directa o inmediata de la verdad — verdad necesaria - de una determinada afirmación. El insight racional indica no sólo que la afirmación es o puede ser verdad sino también por qué lo es y debe ser así (BonJour 2005, 99). No son meras convicciones brutas de la verdad, sino que deben revelar por qué

${ }^{9}$ Explica su teoría en BonJour (I998, 8-11 y 100-13). También Plantinga (I993, 106): "Todo lo que sabemos a priori es necesariamente verdadero"; y Bealer (1999, 30): "podemos tener un conocimiento a priori de $p$ solo si 'se presenta como necesario"'.

${ }^{10}$ BonJour entiende por experiencia no sólo los casos paradigmáticos sino también el ser consciente de los propios pensamientos, sensaciones y otros estados mentales deberían contar como variedades de experiencia, y las razones que ese tipo de experiencias proporcionan debieran contar como empíricas. (La introspección es un tipo de experiencia) (Bonjour 2005, 99). Ej. Cogito cartesiano. 
determinada afirmación es verdadera. Para BonJour, esta explicación surge de la captación directa de cómo se relaciona la conclusión con las premisas y de cómo la validez fluye a través de ellas (BonJour 2005, 100). Así es como deben entenderse los insights lógicos, dice BonJour; deben construirse, además, noproposicionalmente $^{11}$. El intentar hacerlo proposicionalmente conlleva un círculo vicioso como L. Carroll (1895) puso de relieve. Esto es, cuando aceptamos la verdad de la conclusión de un argumento deductivo, hemos de aceptar la verdad de las premisas y la validez del argumento. Eso supone aceptar una nueva premisa: la validez del argumento ("si la premisa A y la premisa B son verdaderas y $\mathrm{C}$ se sigue de $\mathrm{A}$ y $\mathrm{B}$, entonces $\mathrm{C}$ debe ser verdadera” puede considerarse una nueva premisa). Pero ahora tenemos un argumento nuevo, que consta de $\mathrm{n}+1$ premisas. Si aceptamos la validez de este nuevo argumento, entonces tendremos un nuevo argumento, que constará de $\mathrm{n}+2$ premisas y así ad infinitum. Una vez se aprecia la necesidad de aceptar esta concepción no-proposicional del insight para el caso de la inferencia deductiva, es fácil extender este tipo de insight a otros ámbitos.

Para BonJour (2005, 100) hay razones que son genuinamente a priori y que podemos encontrar analizando ejemplos de la matemática y la lógica, algunos de los cuales tienen carácter no-proposicional ${ }^{12}$, como los siguientes:
a) $2+3=5$
b) todos los cubos tienen 12 aristas
c) para toda proposición $\mathrm{P}$ y $\mathrm{Q}$, si es verdadero que $\mathrm{P}$ o $\mathrm{Q}$ y es falsa que $\mathrm{P}$, en- tonces es verdadero que $\mathrm{Q}$

Si entendemos y pensamos con atención cualquiera de estas proposiciones, podremos ver o captar inmediatamente que deben ser verdaderas, y verdaderas en todo mundo o situación posible. Precisamente, este tipo de captar o ver constituye una buena razón, una razón que se impone, para creer que las cosas son así. En este sentido, el insight no depende de la experiencia. Estos

${ }^{11}$ Este aspecto que considera el carácter no-proposicional de los insights lógicos y que había sido objeto de la crítica de Boghossian (200 г B) no era del todo claro en BonJour (I998), [como el mismo BonJour reconoce $(2005,105)$ y también en la réplica a Boghossian (BonJour 200IB)].

12 " [...] Sugiero que el insight lógico relevante debe interpretarse como de carácter no-proposicional, como una captación directa de la forma en que la conclusión se relaciona con las premisas y fluye de manera válida de ellas. Y una vez que se aprecia la necesidad de esta concepción noproposicional de un insight a priori en el contexto de la inferencia deductiva, me parece, de hecho, plausible extenderla también a muchos otros casos; en particular, parece plausible considerar que los insights más fundamentales relativos a cada uno de los ejemplos que se enumeran en la siguiente sección, tienen carácter no-proposicional' (BonJour 2005, 100, cursivas mías). BonJour remite en nota a (BonJour I 998, 142-7). 
ejemplos evidencian la existencia de razones a priori y de la justificación y conocimiento a priori. Para rechazarlos habría que dar una razón alternativa que los hiciera depender de la experiencia (BonJour 2005, 100-1).

Como vemos, los ejemplos $a$ y $b$ de BonJour, son ejemplos de lo que Kant (1787) denominaba juicios sintéticos a priori. Tanto $a$ como $b$ y $c$ contienen conocimiento inferencial que BonJour justifica recurriendo a un elemento no-proposicional y no-inferencial como es el insight. Además de estos ejemplos, BonJour señala otros argumentos como la relación entre la experiencia y ciertas creencias que parecen intuitivamente justificadas por la experiencia. En todo caso, cualquier explicación de la fuerza justificadora de la experiencia ha de hacer la siguiente distinción: hay creencias que se justifican directamente y hay creencias que se justifican indirectamente. La cuestión compleja es la de trazar la línea que las distingue. Según un enfoque fundacionista, las creencias justificadas directamente son aquellas justificadas por el contenido de la experiencia sin necesidad de razonamiento. Pero hay una larga lista de creencias empíricas no justificadas directamente a través de la experiencia, sino indirectamente, como las creencias acerca del pasado no observado; creencias acerca de situaciones no observadas en el presente o creencias acerca del futuro, entre otras. La experiencia puede justificar tales creencias no directamente. La única manera de que la experiencia directa proporcione evidencia para las creencias anteriormente señaladas, es que dispongamos de una buena razón a priori (anterior desde el punto de vista lógico) para una creencia condicional (BonJour 2005, 102). Esto es, el antecedente de ese condicional tendría una conjunción de creencias para las cuales disponemos de evidencia directa, y en el consecuente estaría la creencia indirecta a justificar. Ahora bien, qué razón tenemos para creer en ese condicional. Si todas las creencias para cuya justificación disponemos de evidencia directa están en el antecedente del condicional, y si el consecuente va más allá de lo directamente observado entonces, la experiencia no puede proporcionar una evidencia directa para poder considerar verdadero el condicional (la evidencia indirecta involucraría a su vez otro condicional). Por tanto, la justificación de este tipo de condicional ha de ser total o parcialmente a priori (BonJour 2005, 102-3).

Para BonJour, si no podemos justificar los principios fundamentales del razonamiento, entonces tampoco podemos usar reglas lógicas básicas. Y, cualquier postura que niegue la existencia de razones a priori difícilmente podrá dar cuenta de modo satisfactorio de la capacidad de razonar. El punto fundamental es que una transición razonada o argumentada de un conjunto de premisas a una conclusión depende, de nuevo, de que haya una buena razón para pensar que una afirmación condicional es verdadera (el condicional que 
tiene a la conjunción de las premisas como antecedente y a la conclusión como consecuente). Que tal condicional sea verdadero (o probablemente verdadero) no es algo que se pueda establecer a partir de la experiencia sino a través de un proceso de razonamiento.

Como vemos, el problema se ha desplazado ahora al problema de la naturaleza de la inferencia. BonJour está asumiendo que toda inferencia o razonamiento es un proceso mental. Y la evaluación de la corrección de ese razonamiento es a priori. Para BonJour, por consiguiente, si nunca tenemos razones a priori para pensar que, si una afirmación es verdad, otras también deben ser verdad, entonces no hay nada que pueda ser considerado como razonamiento cogente. El rechazo de las razones a priori es lo mismo que un "suicidio intelectual" (BonJour 2005, 103).

En resumen, BonJour admite las evidencias racionales. El conocimiento lógico es parte del conocimiento a priori (por los argumentos expuestos anteriormente) y, si se quiere evitar la circularidad, la única opción es apelar a los insights lógicos o a la intuición (que para BonJour no son equivalentes). El insight racional es fuente genuina de justificación, aunque pueda ser falible ${ }^{13}$. Los insights lógicos se deben construir no-proposicionalmente. El intentar hacerlo proposicionalmente conlleva un círculo vicioso. Así pues, los insights lógicos deben entenderse como una captación directa de cómo se relaciona la conclusión con las premisas y de cómo "la validez fluye a través de ellas" (BonJour 2005, 100). En definitiva, insight es el acto intelectual de captar las conexiones necesarias y se caracteriza por ser directo, inmediato, no-discursivo, no-proposicional.

\section{\$3.2 Problemas del insight}

Contra propuestas como la de BonJour, se han planteado varias objeciones. Entre otras, para Boghossian (2000; 200 IA; 200 I B; 2003) el insight racional constituye una explicación poco económica y no necesaria. La dificultad principal que señala Boghossian es que el insight racional no evita el problema de la circularidad, ya que se presupone la habilidad de inferir justificadamente de acuerdo al MPP que es la que tienen que explicar (Boghossian 2003A, 234). Boghossian argumenta que nuestras prácticas deductivas no pueden estar basadas en conocimiento proposicional por dos razones. En primer lugar,

${ }^{13}$ La falibilidad no es un problema, pues, para el conocimiento a priori. La mayoría de errores se pueden corregir a través de un cuidadoso y ordenado examen de los vínculos o conexiones y, aun cuando no fuera posible un modo de corregir sistemáticamente los errores, no sería motivo suficiente para abandonar el 'insight a priori" "sin el cual todo razonamiento y crítica sería imposible" (BONJour 2005). 
constituye una explicación muy sofisticada, ya que se está exigiendo que hasta los niños tengan creencias sobre la necesidad o la consecuencia lógica. En segundo lugar, y sobre todo, por el problema, que hemos indicado arriba, de la regresion al infinito que Carroll (I 895) puso en evidencia (Boghossian 200IB, 638).

Un problema añadido que se le presenta a los defensores de la intuición es el de explicar cómo un acto cognitivo es capaz de producir "immediate knowledge of the modal properties of properties" (Boghossian 200 I B, 635): Según Boghossian, parece extraño que apelemos a un tipo natural de insight no-discursivo, "sin raciocinio" (non-ratiocinative) que pueda revelar, inmediatamente y sin la ayuda de razonamiento, que todas las instancias del MPP son preservadoras de verdad (Boghossian 200 i B, 635-6). Así, el problema se presenta al explicar cómo es el acceso cognitivo. No es posible establecer una analogía con la percepción puesto que estamos tratando con objetos abstractos (Boghossian 200 i B, 636) y no podemos basarnos en relaciones causales. No obstante, BonJour sale al paso de esta objeción, ya que basa la idea de una justificación epistémica a partir de la idea de razón a priori que no depende de la experiencia ni de mediación causal, sino sólo "del pensamiento puro o razón" (BonJour I 998, 11).

La objeción de Boghossian a BonJour es que este minimiza la analogía con la percepción y está proponiendo una concepción de contenido mental aristotélica: "[...] de acuerdo a la cual, cuando pensamos en una propiedad dada, como la triangularidad, por ejemplo, esa misma propiedad es puesta como una instancia o evidencia concreta en nuestro pensamiento. Finalmente, BonJour sugiere asimilar el insight racional a una forma de examen introspectivo de ese contenido de pensamiento" (Boghossian 2003, 231). De modo que, en el caso del insight racional, aunque BonJour niega que la justificación requiere una relación causal, si ponemos el acento en el primer término (el insight entendido como aprehensión), entonces la respuesta será causalmente condicionada por los eventos que comprenden los objetos "instanciados" (BonJour I998, 159-60). Por otra parte, si el acento se pone en la razón $a$ priori, entonces el estado cognitivo que justifica, por ejemplo, la creencia $a$ priori de que $p$, incluye la creencia de que $p$, con lo que caerá en el problema de la circularidad.

Veremos si una teoría como la de Bealer resiste las críticas anteriores.

\section{\$3.3 Bealer: Intuición como "parecer" y evidencia}

Bealer (I 998; 2000) defiende que hay "pareceres intelectuales" (intelectual seemings) que son un cierto tipo de estado de conciencia que justifican las 
creencias a priori en las que se fundan. La intuición, así entendida, se define en analogía con la percepción, proponiendo un cierto estado quasi-perceptivo y se explica a través de un cierto "fiabilismo modal": "un fuerte vínculo modal entre lo que [la intuición] aporta y la verdad" (Bealer i 998, 216). Para ello, se centrará en la noción de una posesión "determinada" (en el sentido de entera, completa) del concepto. Según este análisis, poseer un concepto determinadamente es tener las intuiciones correspondientes que siguen la pista de la verdad, asumiendo de partida la ausencia de perturbaciones cognitivas. Para Bealer $(2002,73)$, lo que justifica es el seguir la pista de la verdad, de tal modo que, si alguien posee un concepto determinadamente, las intuiciones correspondientes son evidencia. Aquí, la intuición se define como el estado mental en el que una persona se encuentra cuando le parece que algo es el caso. Una intuición es, por tanto, una clase genuina de episodio consciente (seeming): Tener una intuición que A es parecerle a uno que A. Este tipo de parecer es intelectual, nosensorial o introspectivo (o imaginativo).

La intuición, además, es un "dato de la razón" y no un "dato de la experiencia”. Por ejemplo, al considerar una de las leyes de Morgan, en principio no nos parece ni verdadera ni falsa, sin embargo, "después de un momento de reflexión, sucede algo nuevo: de repente parece verdad" (BEaler 2000, 3). Según Bealer, las intuiciones relevantes para las disciplinas a priori son las intuiciones racionales que se presentan como necesarias, por ejemplo: "si P entonces no no P", esta verdad se presenta como necesaria, parece que no podría ser de otro modo. No obstante, como Martínez-Vidal (2016) ha objetado, si consideramos el caso de un lógico intuicionista y otro clásico, en el caso del concepto asociado a la negación, mientras que para el primero resultará intuitivamente evidente que de $\neg$ P no se sigue P, para el clásico resulta intuitivamente evidente que de $\neg \neg \mathrm{P}$ se sigue $\mathrm{P}$; de modo tal que el concepto de negación que se tenga será el que va a determinar la intuición derivada de la posesión del concepto; así pues, según la teoría de Bealer no lograríamos establecer entre ambos, cuál de los dos es el concepto correcto (tal vez porque no haya uno que sea el concepto correcto de negación en términos absolutos). Si la intuición depende en Bealer de la posesión determinada de un concepto, parece que defensores de la noción clásica de negación y defensores de la intuicionista no se entenderían, aunque cualquiera de ellos coincide con el otro acerca de lo que se sigue si el concepto en uso es el de la negación clásica o el de la intuicionista. Fenomenológicamente, tanto al lógico como al matemático, un enunciado se les presenta como siguiéndose necesariamente de las premisas en la lógica interna a determinado sistema lógico. En el caso de las inferencias básicas, si consideramos que el significado de las expresiones lógicas está dado por las reglas básicas, entonces aceptar que el significado viene dado por la regla es previo a ver regla 
alguna (las básicas también) como válida; las reglas (o las condiciones de verdad o de prueba de las expresiones lógicas) tienen que asumirse como punto de partida. Una vez asumidas, todos podrían estar de acuerdo en qué se sigue de qué, según esta o aquella lógica, y en que los axiomas lógicos son verdaderos (Martínez-Vidal 20i6). Si el problema es la validez de una regla lógica al margen de su aplicación, entonces la explicación de Bealer podría funcionar.

Por otra parte, según Bealer, la intuición es distinta de otras actitudes proposicionales (los juicios, adivinanzas, corazonadas), y del sentido común. Es una actitud proposicional sui generis (BEALER 2002, 74): "Mi punto de vista es, sencillamente, que el parecer intelectual (intuición) es, como el parecer (seeming) sensorial, solo una actitud proposicional primitiva más" (BEALER 2000, 4).

Ahora bien, las paradojas lógicas y otras antinomias han demostrado que ciertas intuiciones pueden ser inconsistentes. Precisamente, la existencia de las paradojas (como, por ejemplo, la paradoja del mentiroso) nos lleva a pensar que las teorías no-falibilistas de la intuición no son tan infalibles. La paradoja del mentiroso muestra que, o bien alguna de nuestras intuiciones de una teoría de verdad ingenua, o bien alguna o algunas de nuestras intuiciones acerca de la lógica clásica, dice Bealer, deberían ser erróneas o reportadas erróneamente. Sin embargo, esto pone en evidencia, más bien, que hay coherencia en nuestras intuiciones elementales concretas, entre otras cosas, y que el equilibrio de dicha coherencia es un hecho general acerca de la cognición humana (BEALER 2000, 4). La conclusión de Bealer es que no podemos evitar racionalmente la tesis de que las intuiciones son evidencia no cancelable.

Sin embargo, una alternativa racional sería la de optar por una noción de justificación más débil, la habilitación (entitlement), por lo que sí podemos evitar racionalmente la tesis de que las intuiciones son evidencia. Esto abre la posibilidad de considerar que la mejor teoría del conocimiento lógico básico es la que opta por defender una debilitación de la noción de justificación. Un tipo de garantía racional no-evidencial, como la de Wright (2004A; 2004B), se puede considerar solo un primer momento, por ejemplo, en la justificación del conocimiento lógico, es decir, una habilitación al uso de la regla básica de inferencia, y ulteriormente podría ratificarse con una justificación del conocimiento de la validez de dicha regla, como veremos. El problema para Bealer es que, al asociar las intuiciones a la evidencia, provoca que se genere el problema de toda justificación epistémica fuerte o evidencialista.

En efecto, Bealer sostiene que el único modo de responder adecuadamente a la pregunta por qué las intuiciones son evidencias es la basada en la verdad: "algún tipo de explicación basada en la verdad (es decir, fiabilista)" (BeALer 2000, 7). En este caso, el fiabilismo no se asocia al conocimiento o a la justificación, sino a la evidencia; pero no como una teoría general de la evidencia, ya 
que las fuentes no básicas de evidencias están sujetas a muchos contraejemplos. Una fuente básica de evidencia fiable es aquella que tiene un vínculo apropiado a la verdad. Y algo es una fuente no básica relativa a un sujeto dado únicamente si a través de la mejor teoría comprehensiva basada en las fuentes básicas de evidencia del sujeto, tiene un vínculo fiable a la verdad (BEALER 2000, 8). Según la tesis tradicional, la experiencia y la intuición fenoménicas son fuentes básicas y las demás son fuentes no-básicas. Para Bealer, el fiabilismo debe limitarse a las primeras. El fiabilismo modal proporciona una explicación natural en virtud del vínculo modal entre estas fuentes y la verdad. Este vínculo entre intuiciones y verdad es consecuencia de lo que, por definición, es comprender los conceptos involucrados en nuestras intuiciones. Así, según su teoría de la posesión del concepto (Bealer 2000, 10), llamamos ordinariamente "comprensión de un concepto" ${ }^{14}$ a poseer un concepto en sentido pleno, completo, fuerte (BEALER 2000, 11). Por tanto, según Bealer, el fiabilismo modal junto al análisis de posesión de un concepto, explican el vínculo entre las fuentes básicas y la verdad.

Así, si lo que le parece intelectualmente a un sujeto tiene el vínculo modal indicado a la verdad, entonces no puede estar equivocado con respecto a lo que le parece reflexivamente que es el contenido de su experiencia fenoménica. No obstante, se podría considerar que, si cambia el modo de posesión de un concepto, no necesariamente por ello va a cambiar la intuición aumentando la comprensión, ya que el modo de posesión podría cambiar, aunque sin implicar necesariamente que haya más comprensión o que la comprensión sea más correcta. Por ejemplo, una persona que ha hecho un curso introductorio de lógica puede pasar de poseer de modo débil el concepto de 'corrección' a llegar a poseerlo determinadamente y saber aplicarlo a inferencias correctas; aunque a la vez, puede no haber llegado a saber que la corrección se considera una propiedad metalógica. Veamos ahora otras objeciones.

\section{\$3.4 Problemas para las teorías basadas en la intuición}

Algunos de los problemas que se les plantean a estas teorías son: en primer lugar, tenemos que ser capaces de explicar cómo la mera comprensión de una proposición puede conferir una capacidad de tener intuiciones fiables, ya que es algo que no se puede dar simplemente por sentado, o explicarlo tomando las mismas intuiciones que constituyen la comprensión de la que se dicen que son una manifestación (Boghossian 2009, 61). En "Inference and Insight", Boghossian (200IB) argumenta, además, que las justificaciones fiabilistas no consiguen explicar el vínculo entre la responsabilidad de un individuo y su práctica cognitiva.

\footnotetext{
${ }^{14}$ Bealer usa el término técnico "poseer un concepto determinadamente" = comprensión de un concepto (posesión de un concepto en el sentido pleno).
} 
Generalmente, se establece una analogía con la visión y se supone que, así como la vista responde fiablemente al sujeto que percibe con normalidad objetos en el ambiente que le rodea, la intuición responde fiablemente a la validez de la lógica básica; sin embargo, mientras que en el caso de la percepción se puede dar una explicación científica de dicho conocimiento, de la intuición no. De modo semejante, Williamson (2007) objeta a teorías como la de Bealer, que defienden las intuiciones racionales como pareceres intelectuales, que tales tipos de "pareceres" carecen de la rica fenomenología de la que, en cambio, sí gozan los pareceres perceptivos (Williamson 2007, 216-7).

En el caso de la intuición lógica, es necesario, por tanto, explicar en qué consiste una facultad quasi-perceptiva de la intuición lógica y cómo esta facultad justifica las creencias lógicas básicas.

En ese sentido, Wright (200 IA, 45) distingue entre justificar las reglas involucradas y verificar que dichas reglas han sido correctamente aplicadas en una instancia dada. Cuando se trata de esto último, un tipo de juicio que se efectúa de modo plenamente consciente de las reglas de inferencia implicadas es un juicio acerca de la forma lógica. Pero este tipo de juicios, dice Wright, no son inferenciales sino directamente comprensivos. Así, la intuición no se puede entender como algo perceptivo literalmente, ya que en los juicios que están contenidos y justificados en los casos en los que la inferencia se realiza en el pensamiento puro, sin ningún tipo de representación física escrita o hablada, en esos juicios no hay objeto de percepción literal. Los juicios básicos de la forma lógica son a priori, pueden ser justificados por pura reflexión (WRIGHT 200IA, 45). Wright caracteriza, así, un tipo de creencias que son justificadas no-inferencialmente, son básicas y a priori, y están implicadas necesariamente en toda inferencia que sea plenamente consciente. Los juicios básicos de la forma lógica los representamos esquemáticamente a partir de oraciones del lenguaje. El problema de querer aplicar esta justificación no-inferencial a las reglas básicas de inferencia es que las creencias acerca de la forma lógica son particulares, se refieren a una proposición determinada, mientras que la creencia de que el MPP es correcto, es la creencia de que toda instancia posible de MPP con premisas verdaderas tiene conclusión verdadera:

Esta generalidad hace que, además, sea inverosímil pensar que tales reglas tienen una justificación (cuasi-) perceptiva. Uno podría (cuasi-) percibir que un objeto particular de atención es así y así, pero ¿cómo puede uno (cuasi-) percibir que todos los objetos de un cierto tipo son así y así? ¿Acaso la generalidad del contenido de la supuesta (cuasi-) percepción simplemente no deja de lado alguna analogía seria con la percepción? (WRIGHT 200 IA, 46) 
Wright (200 IA, 81-2, nota 5) indica que el conocimiento de la validez de una regla de inferencia conlleva dos cosas, i) la identificación de la instancia y ii) la fundamentación de que esa instancia es válida.

A nuestro parecer, la objeción de Wright es la mayor dificultad a la que se enfrentan las teorías de la intuición y cualquier justificación no-inferencial para el caso de la lógica, porque se enfrenta al problema de cómo pasar de una regla particular a la generalidad para todas las instancias de la regla. ${ }^{15}$

Desde la noción de intuición de Bealer, no se puede salvar tampoco este problema porque se basa en una teoría de la justificación epistémica en sentido fuerte, donde las intuiciones son evidencias. Si se pone el acento en los pareceres intelectuales, a partir de la analogía con los pareceres perceptivos, tendríamos conocimiento quasi-empírico cuya certeza racional provendría de su vínculo modal a la verdad, pero si el contenido de la intuición es no-proposicional, no se entiende muy bien cómo sería posible. El problema, desde nuestro punto de vista, es sostener que las intuiciones son evidencia, porque se convierten en el hecho que debe ser a su vez explicado; si la intuición tiene contenido proposicional (en el caso de Bealer por su teoría de la posesión del concepto), se genera circularidad en la justificación, ya que necesitamos una intuición de la intuición que justifique la intuición-evidencia. Como toda justificación en sentido fuerte, se ve abocada a la regresión al infinito o a la circularidad.

No obstante, sería posible respaldar el enfoque de la intuición comenzando por revisar la definición de intuición; en segundo lugar, ajustando la teoría de Bealer, asumiendo que las intuiciones no son evidencias, sino que se apoyan en garantías no-evidenciales que siguen la pista de la verdad. En tercer lugar, habría que sostener que, al contrario que para Bealer, es la comprensión del concepto la que deriva de la intuición.

\section{\$4. ¿INTUICIÓN O GARANTÍA RACIONAL NO-EVIDENCIAL?}

Como hemos visto, según Bealer, una intuición a priori deriva de la comprensión determinada del concepto, y esta es fiable porque sigue la pista de la verdad, hay un vínculo modal entre la intuición y la verdad. Bealer indica que es un "parecer" verdadero: "Por ejemplo, cuando consideras por primera vez una de las leyes de Morgan, a menudo ni parece ser verdad ni parece ser falsa; después de un momento de reflexión, sin embargo, algo nuevo sucede: de repente parece verdadera" (Bealer 2000, 3). Para BonJour, no la reflexión sino

${ }^{15}$ Aunque por ese motivo la vía rápida de solución ha sido clásicamente la intuición: “(...) es partiendo de lo individual como se llega a lo universal. Así pues, debemos tener percepción sensible de estos particulares, y ésta es la intuición." (ARIstóteles, Ética a Nicómaco, Z, 1143b). No obstante, no llega a explicar de un modo viable cómo es ese paso de lo particular a lo universal. 
la captación, el ver (insight) la necesidad de la proposición, es el conocimiento directo e inmediato (y por eso básico).

Además de las descripciones fenomenológicas de BonJour y Bealer, hay metodologías como la de Würzburg Schoo ${ }^{16}$ que permiten una mayor especificación de la intuición. Bühler (VALsiner et al. 2009, 330) define la intuición caracterizándola dentro de los "momento-aha" ("Aha-experience"). Son momentos de sorpresa y comprensión ("Aha-Erlebnis") en los que lo que antes era difuso se vuelve algo concreto y se puede articular, se convierte en algo elaborado sintéticamente a través de asociaciones (VAlsiner et al. 2009, 341). Se trata de una comprensión retrospectiva, que puede ocurrir o no de modo repentino; son -según Bühler- tipos de pensamiento que no tienen imágenes (Valsiner et al. 2009, 330). Tres de los ejemplos paradigmáticos que Bühler señala son: la conciencia de una regla (Das Regelbewusstsein), esto es, el pensar en la forma de una regla o a través de una regla lógica, por ejemplo; es decir, tomar conciencia de cómo resolver un problema a través de un método. En segundo lugar, la conciencia de las relaciones (Das Beziehungsbewusstsein): el tomar conciencia de las relaciones entre pensamientos. Y, el momento-aha ("Aha-moment" o "Aha-Erlebnis") que se caracteriza, según Bühler, en la comprensión entre dos totalidades (zwischen Ganzem und Ganzem), de donde se colige que "(...) 'lo que debe ser comprendido, primero necesita convertirse en un todo' (BüHLER I 908A, 17)" (VALSINeR et al. 2009, 330-1).

Teniendo en cuenta esta caracterización, podríamos perfilar las de BonJour y Bealer. Según BonJour (1998, 106-7), al considerar, por ejemplo, una inferencia, captamos que la conclusión debe ser verdadera si las premisas son verdaderas. Como vemos, en esta caracterización podemos distinguir los tres ejemplos de Bühler: captamos el pensamiento en la forma de la regla, tomamos conciencia de las relaciones entre pensamientos y se produce la comprensión a partir de la consideración de la inferencia tomada como un todo. La diferencia es que para BonJour se trata de una "aha-experience" representacional y de modo semejante, para Bealer. Se trata de quasi-percepciones. En cambio, Bühler entiende que este tipo de pensamientos no necesariamente conlleva imágenes. Recordemos que dos de las fuentes principales de conocimiento básico, según el fundacionismo, son la intuición y la experiencia y se consideran principales porque ambas son inmediatas y no-inferenciales lo cual permitiría evitar la circularidad en la justificación (aunque ya hemos visto que no lo consiguen). Tanto la intuición como la experiencia tienen contenido representacional, no-conceptual, por lo tanto, son no-proposicionales ${ }^{17}$. En cambio, para

${ }^{16}$ Würzburg School methodologies: "Aha-experience" (VALSINER et al. 2009, 330-1).

${ }^{17}$ Aunque hay autores como McDowell (1994) que defienden que la experiencia tiene contenido conceptual. 
Bühler, la intuición o 'aha-experience' tiene contenido conceptual. El problema es si ese contenido es además proposicional, pues entonces, caemos de nuevo en la circularidad que queríamos evitar.

Aquí habría que distinguir entre captar que una cosa "se sigue" inferencialmente de otra, y captar un pensamiento. Para Frege, captar un pensamiento no es intuir, es pensar. Según Frege, captamos pensamientos y los podemos reconocer como verdaderos: " $\mathrm{Si}$, por ejemplo, capto el pensamiento que expreso en el teorema de Pitágoras, la consecuencia puede ser que lo / acepte como verdadero y, además, lo aplique al tomar una decisión que dé lugar a una aceleración de las masas" (Frege i 91 8-I919, \$77 [trad. esp.: Frege 2013, 224]).

Teniendo en cuenta estos elementos y a la luz del parágrafo final de Der Gedanke de Frege, se podría intentar matizar una definición de intuición. Intuir, así como imaginar ${ }^{18}$, por ejemplo, son capacidades que se ejercitan con la facultad de la inteligencia, son actos de intelección; así como uno imagina una determinada situación, también piensa-intuye una forma/regla lógica, "ve" o "se da cuenta" cómo una cosa se sigue de otra, toma conciencia de las relaciones entre pensamientos. Tomando un ejemplo de Bealer, puedo estar delante de una ley lógica como la ley de De Morgan sin "parecerme" ni verdadera ni falsa; hasta que en un momento "me doy cuenta", "capto" cómo están relacionados sus elementos entre sí, el pensamiento que expresa la ley de De Morgan (que no tiene por qué ser inmediato, puede llevar más o menos tiempo). De este modo, se hace presente un pensamiento bajo un aspecto inteligible que es posible distinguir de una imaginación. Así, en un primer momento, captamos cómo en la regla del MPP, por ejemplo, la conclusión se sigue de las premisas. El momento posterior comprensivo de esa regla ${ }^{19}$ (captar el pensamiento de la validez del MPP) justifica el conocimiento de que esa instancia es válida. Se trata de dilucidar cuál es la cuestión más básica, si la práctica o uso de una regla, captar cómo una cosa se sigue de otra, o captar el pensamiento de la validez de la regla. En otras palabras, ¿"intuir" es algo que hacemos o es algo que nos pasa? Desde el enfoque que aquí sostenemos, intuir es algo que hacemos, es

${ }^{18}$ Williamson (2007), a propósito del conocimiento de la modalidad metafísica, sostiene que el "concebir" o "imaginar", el ejercicio imaginativo podría considerarse un tipo de razonamiento (Williamson 2007, 143). Según Williamson, la justificación y las normas epistémicas deben ser entendidas de una manera radicalmente externista. Los experimentos mentales y los métodos filosóficos no se basan en la intuición racional o en la competencia conceptual, sino en nuestra imaginación empíricamente formada.

${ }^{19}$ En Boghossian (200IB) se encuentran también dos momentos, pero caracterizados de modo distinto: en primer lugar, una disposición a razonar de acuerdo a la regla de inferencia MPP que habilita a priori a realizar dicha inferencia. En segundo lugar, la creencia de que el MPP es válido, que justifica el conocimiento lógico. Boghossian (200 I B, 633) señala que la cuestión de la práctica es más básica que la cuestión acerca de la creencia. Esa práctica resultará ser un razonamiento "ciego". 
poner en relación las partes considerando el conjunto o el sistema, pero no como un proceso (relacionar o inferir), sino como un acto (captar la relación inferencial). En ese sentido, captar o intuir una inferencia básica es una acción mental no-inferencial y no-proposicional. No obstante, si entendemos así la intuición, dejamos de considerarla una fuente básica ya que parece apoyarse en una cierta garantía racional.

En efecto, Burge (1993), sostiene que, en ausencia de razones contrarias, un sujeto está racionalmente legitimado ${ }^{20}$ a confiar en el razonamiento deductivo, dado que una de las funciones primarias de la razón es la de presentar la verdad (Burge 1993, 475). Este tipo de justificación, en sentido débil, que es fundamentalmente a priori, no deriva de la comprensión conceptual, sino que el entendimiento es epistémicamente básico (BURGE 1993, 479). O, dicho de otro modo, la legitimación fundamental para aceptar algo que se presenta como verdadero, deriva en última instancia del entendimiento (BURGE I993, 483), ya que, la inteligibilidad es a priori un signo de racionalidad prima facie (Burge I993, 488). Es decir, antes de cualquier posibilidad de justificación, aquello que se nos presenta como verdadero debe ser inteligible. Esa inteligibilidad es de la que parte Burge como elemento necesario y previo de toda posible justificación. En el caso del conocimiento lógico, al lógico se le presenta un enunciado como siguiéndose necesariamente de las premisas en la lógica interna a determinado sistema lógico. Esta presentación, es el modo de entender la intuición para Bengson (20 I 5). Desde nuestra perspectiva, la intuición no es tanto la presentación cuanto el poner en relación concibiendo la lógica propia interna del sistema.

No obstante, persistiría la crítica de Wittgenstein:

No hay acto de captación, de intuición, que nos haga usar la regla como lo hacemos en el punto particular de la serie. Sería menos confuso llamarlo un acto de decisión, aunque también esto es desorientador, pues no tiene que realizarse nada parecido a un acto de decisión, sino tal vez solo un acto de escribir o hablar. Y la falta que tendemos a hacer aquí y en mil casos semejantes está

${ }^{20}$ Según Casullo, la noción de entitlement no es nueva desde el punto de vista conceptual: “¿Es la habilitación (entitlement) un nuevo concepto epistémico? No. La habilitación es una especie de apoyo epistémico positivo que es (solo) moderadamente externo. (...), tanto Alston como Burge sostienen que existe una especie de apoyo epistémico positivo que es suficiente para el conocimiento y que es solo moderadamente externo (y solo débilmente interno). (...) Burge, por otra parte, desea reservar el término 'justificación' para referirse exclusivamente a una especie particular moderadamente interna de apoyo epistémico positivo. Por lo tanto, debe introducir el nuevo término 'garantía' para referirse al género y el nuevo término 'habilitación' para referirse a las especies débilmente internas del género. La innovación es terminológica más que conceptual" (CAsullo 2007, 278). 
marcada por la palabra "hacer" tal como la hemos usado en la frase: "No hay acto de captación que nos haga usar la regla tal como lo hacemos", porque hay una idea de que "algo tiene que hacernos" hacer lo que hacemos. Y esto vuelve a enlazarse con la confusión entre causa y razón. No necesitamos tener razón alguna para seguir la regla tal como lo hacemos. La cadena de razones tiene un límite (Wittgenstein i 958, 143, “Brown Book” secc. II \$42 [trad. esp.: WittgensTEIN 2OI4, 191])

Para salvar esta objeción, consideramos con Wright (2014) que las inferencias básicas son acciones mentales básicas, de modo que tales inferencias tienen que asumirse como punto de partida, en un sentido semejante al que encontramos en Wittgenstein. En ese caso, la intuición es posterior, no es lo que justifica. Partimos de una confianza racional. Según Wright, cuando un sujeto tenga los recursos para preguntar si una inferencia particular es válida, una garantía que permita confiar en su validez (WRIGHT 200IA, 80) será suficiente para justificar la inferencia. En ese sentido, es una justificación en sentido débil, ya que la garantía racional es sólo una habilitación al uso de la regla básica de inferencia que no exime de una posterior justificación del conocimiento de la validez de dicha regla. Se trata de una especie de soporte epistémico positivo, suficiente para el conocimiento, y que se explica, a nuestro juicio, desde una teoría de la justificación moderadamente externista y solo débilmente internista (CASullo 2007, 278). Se trata de una justificación no-inferencial de las reglas generales y, en los casos suficientemente simples, de la validez de las inferencias particulares. Así entendida, la justificación del conocimiento lógico básico es una garantía que, en última instancia, no está mediada por inferencia alguna ni procede de una intuición.

\section{\$5. ConCLUSIÓN}

En este trabajo hemos presentado las dificultades con las que se encuentran aquellos que tratan de justificar el conocimiento lógico básico no-inferencialmente recurriendo a la intuición. Esta opción podría en principio evitar el círculo que se genera al establecer que los principios de la lógica se conocen a partir de inferencias. De tal modo que, si una inferencia proporciona conocimiento a priori, es debido a una evidencia racional directa. La hemos descartado porque a la hora de explicar el conocimiento intuitivo de las reglas lógicas básicas, aunque podríamos establecer una definición que haga ver cómo actúa la intuición, en el caso de las inferencias básicas, intuir no es el elemento activo necesario para la justificación ya que se apoya en una confianza racional. De 
ahí que, el camino a tomar es debilitar la noción de justificación en sentido de una "habilitación".

\section{Agradecimientos}

Agradezco a los profesores Concha Martínez Vidal y Xavier de Donato Rodríguez que tuvieron la paciencia de leer numerosas versiones previas del trabajo. Sus comentarios son siempre una ayuda y un estímulo. 


\section{BIBLIOGRAFÍA}

Aristóteles, Ética a Nicómaco, Madrid: Gredos, I988.

Audi, R. 200I, The Architecture of Reasons, Nueva York: Oxford University Press.

Bealer, G. I998, "Intuition and the Autonomy of Philosophy", en De Paul and

Ramsey (ed.), Rethinking Intuition, Lanham, MD: Rowman and Littlefield.

Bealer, G. I999, "A Theory of the A Priori", Philosophical Perspectives, 13: 29-55.

Bealer, G. 2000, "A Theory of the A priori", Pacific Philosophical Quarterly, 81: 1-30.

Bealer, G. 2002, "Modal Epistemology and the Rationalist Renaissance", en T.

Gendler and J. Hawthorne, Conceivability and Possibility. Oxford: Oxford University Press, 71-125.

Bengson, J. 201 5, "The Intellectual Given”, Mind, 124, 495: 707-60.

Bermejo, L. 20 i i, "Circularidad", en L. Vega y P. Olmos (ed.), Compendio de Lógica, Argumentación y Retórica, Madrid: Trotta, 94-5.

Boghossian, P. A. 2000, "Knowledge of logic", en P. A. Boghossian, New Essays on the A Priori, Oxford: Oxford University Press, 229-54.

Boghossian, P. A. 200 in, "How are Objective Epistemic Reasons Possible?" Philosophical Studies, 1/2, 106: 1-40.

Boghossian, P. A. 200 Iв, "Inference and Insight", Philosophy and Phenomenological Research, LXIII, 3: 633-40.

Boghossian, P. A. 2003, "Blind Reasoning", Proceedings of the Aristotelian Society, Supl., 77: 225-48. En Boghossian, P. A. 2008, Content and Justification: Philosophical Papers, Oxford: Oxford University Press, 250-67.

Boghossian, P. A. 2008, Content and Justification: Philosophical Papers, Oxford: Oxford University Press.

Boghossian, P. A. 2009, "Virtuous Intuitions: Comments on Lecture 3 of Ernest Sosa's 'A Virtue Epistemology”, Philosophical Studies, 144 (1): 111-9.

Boghossian, P. A. y Peacocke, C. 2000, New Essays on the A Priori, Oxford: Oxford University Press.

BonJour, L. I985, The Structure of Empirical Knowledge, Cambridge: Harvard University Press.

BonJour, L. 1998, In Defense on Pure Reason: a Rationalist Account of A Priori Justification, Cambridge: Cambridge University Press.

BonJour, L. 200 ia, "Precis of In Defense of Pure Reason", Philosophy and Phenomanological Research, 63: 625-31.

BonJour, L. 200 I в, "Replies”, Philosophy and Phenomenological Research, 63: 673-98.

BonJour, L. 2005, "Is there A Priori Knowledge? In Defense of the A Priori", en E. Sosa y M. Steup, Contemporary Debates in Epistemology, Cambridge, MA: Blackwell Publishers, 98-105. 
Brandom, R. B. 2000, Articulating Reasons: An introduction to Inferentialism, Harvard University Press.

Burge, T. I993, "Content Preservation", The Philosophical Review, 102: 457-88.

Burge, T. 2005, Truth, Thought, Reason: Essays on Frege, Oxford: Oxford University Press.

Carroll, L. I895, What the tortoise said to Achilles, Mind, 4 (14): 278-80.

Casullo, A. 2007, "What Is Entitlement?" Acta analytica, 22: 267-79.

De Paul, M. (ed.) 200 I, Resurrecting Old Fashioned Foundationalism, Lanham: Rowman and Littlefield.

Descartes, R. I70 i, Reglas para la dirección del espiritu, Madrid: Alianza Editorial, $20 I 0$.

Dogramaci, S. 20 i 2, "Apriority”, en D. G. Fara y G. Russell, The Routledge Companion to Philosophy of Language, Nueva York: Routledge, 768-82.

Field, H. 2000, "Apriority as an Evaluative Notion", en P. Boghossian y C. PeaCocke (ed.), New Essays on the A Priori, Oxford: Clarendon Press, 117-49.

Frege, G. I897, "Logic", en Posthumous Writings, H. Hermes, F. Kambartel y F. Kaulbach (ed.) I979, Oxford: Basil Blackwell, 126-51.

Frege, G. i9i 8-i9i9, "Der Gedanke”, en M. Beaney (ed.) 1997, The Frege Reader, Wiley-Blackwell.

Frege, G. 1964, The Basic Laws of Arithmetic, Berkeley y Los Angeles: University of California Press.

Frege, G. I979, Posthumous Writings, H. Hermes, F. Kambartel y F. Kaulbach (ed.), Oxford: Basil Blackell.

Frege, G. 2013, Ensayos de semántica y filosofía de la lógica, Madrid: Tecnos.

García-Carpintero, M. 2005, "Intuiciones y contenidos no-conceptuales", en Grimaltos-Pacho (ed.), La naturalización de la filosofía: problemas y limites, Valencia: Pre-textos, 109-23.

Gibbard, A. I990, Wise Choices, Apt Feelings: A Theory of Normative Judgement, Oxford: Oxford University Press. [Reedición 2002]

GöDel, K. I964, “What is Cantor's Continuum Problem?”, en P. Benacerraf (ed.), Philosophy of Mathematics, Englewood Cliffs, 258-73.

Kant, I. 1787 [2007], Critica de la razón pura, Buenos Aires: Colihue.

Leibniz, G. W. I983, Nuevos ensayos sobre el entendimiento humano, Madrid: Editora Nacional.

McDowell, J. I 994, Mind and World, Cambridge, Mass: Harvard University Press.

Martínez-Vidal, C. 2008, "El 'Status' epistemológico de la lógica: verdad y necesidad”, en M. J. Frapolli, Filosofía de la Lógica, Madrid: Tecnos, 83-118.

Martínez-Vidal, C. 20i6, "A vueltas con la intuición y el conocimiento matemático”, en A. Lassalle Casanave y J. Ferreirós (ed.), El árbol de los números. 
Cognición, lógica y práctica matemática, Sevilla: Editorial Universidad de Sevilla, 171-90.

Plantinga, A. 1993, "A Priori Knowledge", en Warrant and Proper Function, Nueva York: Oxford University Press, 102-21.

Plantinga, A. 2000, "Warrant: A First Approximation", en Sosa, E., Epistemology, Oxford: Blackwell, 445-56.

Quine, W. V. 1936, "Truth by Convention”, en W. Quine 1966, The Ways of Paradox and Other Essays, Nueva York: Random House. [2a ed. Cambridge, Mass.: Harvard University Press, 1976.]

Quine, W. V. 1963, "Carnap and Logical Truth", en W. Quine 1966, The Ways of Paradox and Other Essays, Nueva York: Random House.

Sellars, W. 1963, "Empiricism and the Philosophy of Mind", en Science, Perception and Reality, Londres: Routledge and Kegan Paul.

SosA, E. 2007, "Intuitions: Their nature and Epistemic Efficacy", en Grazer Philosophische Studien, special issue: C. Beyer y A. Burri (ed.), Philosophical Knowledge: Its Possibility and Scope, Ámsterdam: Rodopi.

Sosa, E. 2013, "Intuitions and Foundations. The Relevance of Moore and Wittgenstein", en Casullo, A. y Thurow, J. C. (ed.), The A Priori in Philosophy, Oxford: Oxford University Press.

Valsiner, J., Molenaar, P. C. M., Lyra, M. C. D. P. y Chaudhary, N. (ed.) 2009, Dynamic Process Methodology in the Social and Developmental Sciences, Dordrecht: Springer. DOI 10.1007/978-0-387-95922-1.

Williamson, T. 2003, "Understanding and Inference", Proceedings of the Aristotelian Society, Supplementary Volume, LXXVII: 249-93.

Williamson, T. 2007, The Philosophy of Philosophy. Oxford: Blackwell.

Wittgenstein, L. 1958, The Blue and Brown Books, Oxford: Blackwell Publishers Ltd. [Segunda edición I969.]

Wittgenstein, L. 20145', Los cuadernos azul y marrón, Madrid: Tecnos.

Wright, C. 200ia, "On Basic Logical Knowledge", Philosophical Studies, 106: 41-85. [Reimpresión en J. Bermudez y A. Millar (ed.), Reason and Nature, Oxford, Clarendon Press, 49-84.]

Wright, C. 200 I B, Rails to Infinity: Essays in Themes from Wittgenstein's Philosophical Investigatios, Cambridge, Mass.: Harvard University Press.

Wright, C. 2004A, "Warrant for nothing (and foundations for free)?", Aristotelian Society Supplementary Volume, 78 (1): 167-212.

Wright, C. 2004B, "Intuition, Entitlement and the Epistemology of Logical Laws", Dialectica, 58: 155-75.

Wright, C. 20I4, "Comments on Paul Boghossian, 'What Is Inference?", Philosophical Studies, 169: 27-37. 